\title{
Eficácia e Persistência no Solo de Herbicidas Utilizados em PASTAGeM $^{1}$
}

\author{
Efficacy and Persistence of Herbicides in Pasture Soils
}

\author{
SANTOS, M.V. ${ }^{2}$, FREITAS, F.C.L. ${ }^{3}$, FERREIRA, F.A. ${ }^{4}$, VIANA, R.G. ${ }^{5}$, TUFFI SANTOS, L.D. ${ }^{6}$ e \\ FONSECA, D.M. ${ }^{7}$
}

\begin{abstract}
RESUMO - Este trabalho teve como objetivo avaliar a eficácia dos herbicidas 2,4-D + picloram (Tordon), fluroxypyr + picloram (Plenum) e triclopyr (Garlon) no controle de aroeirinha (Schinus terebintifolius) e mata-pasto (Eupatorium maximilianii), bem como a ação residual desses herbicidas no solo. O estudo foi conduzido em pastagem estabelecida de capim-gordura (Melinis minutiflora). O delineamento experimental foi em blocos casualizados, com quatro repetições e 13 tratamentos, distribuídos em esquema fatorial $(3 \times 4+1)$, sendo duas misturas comerciais e um herbicida, aplicados em quatro doses: 2,4-D + picloram [360 + $96 \mathrm{~g} \mathrm{ha}^{-1} ; 720+$ $\left.192 \mathrm{~g} \mathrm{ha}^{-1} ; 1.080+288 \mathrm{~g} \mathrm{ha}^{-1} ; 1.440+384 \mathrm{~g} \mathrm{ha}^{-1}\right]$; fluroxypyr + picloram [120 + $120 \mathrm{~g} \mathrm{ha}^{-1} ; 240$ + $240 \mathrm{~g} \mathrm{ha}^{-1} ; 380+380 \mathrm{~g} \mathrm{ha}^{-1} ; 480+480 \mathrm{~g} \mathrm{ha}^{-1}$ ] e o herbicida triclopyr $\left(240,480,720\right.$ e $\left.960 \mathrm{~g} \mathrm{ha}^{-1}\right)$, além de uma testemunha sem herbicida. Foram realizadas avaliações de intoxicação na pastagem aos 7 e 15 DAA e de controle das plantas daninhas aos 60 e 180 DAA. O resíduo no solo foi avaliado por meio de bioensaios em vasos, em casa de vegetação, com amostras de solo coletadas nas parcelas experimentais, nas profundidades de 0 a 10 e 10 a $20 \mathrm{~cm}$, aos 10,40,70, 120, 180 e 360 DAA, utilizando-se pepino (Cucumis sativus) como planta indicadora. Controle superior a 90\% de Schinus terebintifolius e Eupatorium maximilianii foi obtido aos 180 DAA, respectivamente a partir de: 2,78 e 2,46 L ha-1 para o Tordon; 1,97 e 2,02 L ha-1 para o Plenum; e 1,23 e 1,44 $\mathrm{L} \mathrm{ha}^{-1}$ para o Garlon. Verificou-se resíduo no solo até os 360 DAA para as misturas comerciais de herbicidas que continham picloram na formulação. Resíduo do herbicida triclopyr foi observado somente nas amostras coletadas aos 10 DAA, indicando curta persistência no solo.
\end{abstract}

Palavras-chave: triclopyr, picloram, fluroxypyr, resíduo, controle, plantas daninhas.

\begin{abstract}
This work aimed to evaluate the efficacy of the herbicides 2,4-D + picloram (Tordon); fluroxypyr + picloram (Plenum) and triclopyr (Garlon) in the control of Schinus terebintifolius and Eupatorium maximilianii, which are important weeds in pastures. The experiment was developed in a pasture of Melinis minutiflora, arranged in a randomized complete block design, with four replications, distributed in a factorial scheme $(3 \times 4+1)$, using two commercial mixtures and herbicide applied at four rates: 2,4-D + picloram $\left[360+96 \mathrm{~g} \mathrm{ha}^{-1} ; 720+192 \mathrm{~g} \mathrm{ha}^{-1} ; 1.080+288 \mathrm{~g} \mathrm{ha}^{-1}\right.$; $1.440+384 \mathrm{~g} \mathrm{ha}^{-1}$; fluroxypyr + picloram [120 + $120 \mathrm{~g} \mathrm{ha}^{-1} ; 240+240 \mathrm{~g} \mathrm{ha}^{-1} ; 380+380 \mathrm{~g} \mathrm{ha}^{-1}$; $480+480 \mathrm{~g} \mathrm{ha}^{-1}$ triclopyr herbicide: 240; 480; 720 and $960 \mathrm{~g} \mathrm{ha}^{-1}$ plus a control without herbicide. Intoxication in the pasture was evaluated at 7 and 15 DAA (Days After Application) and weed control at 60 and 180 DAA. The residue in the soil was evaluated through bioassay, in pots in the greenhouse, with soil samples collected in the experimental plots at depths of $0-10$ and $10-20 \mathrm{~cm}$, 10,40,70,120, 180 and 360 DAA, with Cucumis sativus used as test plant. Controls over $90 \%$ of Schinus terebintifolius and Eupatorium maximilianii were obtained 180 DAA, respectively, as follows 2.78 and $2.46 \mathrm{~L} \mathrm{ha}^{-1}$ on Tordon; 1.97 and $2.02 \mathrm{~L} \mathrm{ha}^{-1}$ on Plenum; and 1.23 and $1.44 \mathrm{~L} \mathrm{ha}^{1}$ on Garlon. Residues in the soil were verified until $360 \mathrm{DAA}$ for herbicide commercial mixtures containing picloram in their formulation. Triclopyr herbicide residue was verified only at 10 DAA, indicating short persistence in the soil.
\end{abstract}

Keywords: Triclopyr, picloram, fluroxypyr, residue, controls, weeds.

Recebido para publicação em 22.11.2005 e na forma revisada em 5.5.2006.

2 Estudante de Mestrado em Fitotecnia, Departamento de Fitotecnia, Universidade Federal de Viçosa - DFT/UFV, $36570-000$ Viçosa-MG, <marciavitori@ hotmail.com>; ${ }^{3}$ Doutor em Fitotecnia - DFT/UFV; ${ }^{4}$ Professor Titular - DFT/UFV; ${ }^{5}$ Estudante de Mestrado em Fitotecnia - DFT/UFV; ${ }^{6}$ Estudante de Doutorado em Fitotecnia - DFT/UFV. ${ }^{7}$ Professor Adjunto do Dep. de Zootecnia - DZO/UFV. 


\section{INTRODUÇÃO}

Uma característica importante da pecuária brasileira é a grande disponibilidade de pastagens, o que possibilita a produção de carne bovina e leite de forma competitiva em termos de custo de produção e qualidade. No entanto, pastagens degradadas, com alta infestação de plantas daninhas, como aroeirinha (Schinus terebintifolius) e mata-pasto (Eupatorium maximilianii), na Zona da Mata de Minas Gerais, constituem-se atualmente em um dos maiores problemas do sistema de produção de bovinos no Brasil.

No manejo de plantas daninhas em pastagens procura-se eliminar os prejuízos por elas causados, resguardando seus aspectos benéficos, sem causar danos às forrageiras, aos animais e ao solo. Dos métodos de controle disponíveis, o químico é empregado com a finalidade de inibir o desenvolvimento e/ou provocar a morte das plantas daninhas; entretanto, este método deve ser associado com outras práticas de controle, sendo a de maior importância o controle cultural (Silva et al., 2002).

Na maioria das vezes, os produtores e até mesmos os técnicos se preocupam apenas com a eficiência do herbicida em eliminar as plantas daninhas problemas e o custo do tratamento. Pouca importância é dada ao comportamento do herbicida no solo. Todavia, é de conhecimento público que alguns dos herbicidas mais usados em pastagem apresentam longo período residual no solo, podendo causar danos a culturas subseqüentes, ou a culturas muito sensiveis a resíduos desses produtos, quando se utiliza a matéria orgânica proveniente de áreas tratadas com esses herbicidas (Silva et al., 2005).

A atividade biológica de um herbicida é função da absorção, translocação, metabolismo e suscetibilidade da planta a este herbicida. Portanto, a dose de um herbicida requerida para o controle de plantas daninhas varia em função da espécie, do estádio de desenvolvimento da planta e de sua atividade metabólica no momento da aplicação (Silva et al., 2005).

O comportamento de um herbicida no solo é influenciado por processos de retenção, transformação e transporte que ocorrem imediata e/ou simultaneamente após sua aplicação (Weber \& Miller, 1989). Esses processos são dependentes de diversos fatores que atuam de forma combinada, destacando-se: o $\mathrm{pH}$, a matéria orgânica, a textura e mineralogia, a temperatura, a umidade e a dose aplicada (Silva et al., 2005). Segundo Oliveira Junior et al., (2001), devido aos vários fatores envolvidos, não é possível predizer o tempo exato que é requerido para a dissipação de um herbicida aplicado ao solo sob um conjunto particular de condições. A meia-vida é um parâmetro comum usado para comparar a persistência de herbicidas no solo. Entretanto, a literatura é carente de informações sobre eficácia, período residual dos herbicidas no solo e, principalmente, recomendações para controle de espécies arbustivas infestantes de pastagens.

Dentre os herbicidas registrados para pastagens no Brasil, destacam-se 2,4-D + picloram, fluroxypyr + picloram e triclopyr (Silva et al., 2005). O picloram se caracteriza por ser um produto extremamente ativo em dicotiledôneas, sendo comum seu uso em misturas com outros herbicidas, como 2,4-D ou fluroxypyr. Apresenta longa persistência no solo, com meia-vida de 90 dias (Rodrigues \& Almeida, 2005), podendo ser encontrado até dois ou três anos após a aplicação em área total (Deubert \& Corte-Real, 1986; WSSA, 1994); possui alta solubilidade em água, pka 2,3; Kow 1,4 a pH 7,0 e 83,2 a pH 1; e Koc médio de $16 \mathrm{~mL} \mathrm{~g}^{-1}$ de solo (Silva et al., 2005). Segundo Inoue et al. (2003), por apresentar longa persistência e baixo Koc, é considerado um herbicida com alto potencial de contaminação de águas subterrâneas. Quanto ao triclopyr, apresenta solubilidade de $430 \mathrm{ppm}$; pka $=2,68$; Kow $=$ 2,64 a pH 5 e 0,36 a pH 7; e Koc médio de $\mathrm{mL} \mathrm{g}^{-1}$ de solo (Rodrigues \& Almeida, 2005); possui meia-vida de 20 a 45 dias, apresentando média persistência no solo, dependendo do tipo de solo e das condições climáticas. Em solos com pH baixo, o seu grau de adsorção é mais elevado e a mobilidade é menor. O fluroxypyr apresenta comportamento no solo semelhante ao de triclopyr, com meia-vida de 34 a 63 dias, dependendo do tipo de solo. O 2,4-D apresenta solubilidade de $600 \mathrm{ppm}$, pka $=2,8$ e Koc médio de $20 \mathrm{~mL} \mathrm{~g}^{-1}$ de solo (Rodrigues \& Almeida, 2005) e persistência curta no solo, com média de 30 dias; é eficiente apenas para 
o controle de plantas daninhas dicotiledôneas anuais, sendo por isso recomendado em mistura com outros herbicidas auxínicos para controle de plantas daninhas em pastagem.

Uma das técnicas mais comuns para identificar e quantificar um herbicida no solo ou na água é por meio de bioensaios utilizando plantas indicadoras que apresentam fácil cultivo, rápido desenvolvimento e alta sensibilidade aos herbicidas avaliados (Nyffeler et al., 1982; Souza, 1999). Segundo Thill (2003), sintomas de intoxicação produzidos nas folhas de várias dicotiledôneas, por herbicidas auxínicos, são facilmente caracterizados e por isso muito usados para detectar resíduos desses herbicidas no solo. Uma das espécies mais utilizadas nesses bioensaios é o pepino (Cucumis sativus).

Os herbicidas auxínicos causam, em espécies sensiveis, intensa divisão celular no câmbio vascular, endoderme, periciclo e floema, com conseqüente formação de tumores no meristema intercalar, aparecimento de raízes aéreas, multiplicação e engrossamento de raízes e caule. Há formação de gemas múltiplas e hipertrofia das raízes laterais, sendo o encurtamento do tecido internerval e a epinastia os sintomas mais evidentes nas espécies dicotiledôneas (Silva et al., 2005).

Este trabalho teve como objetivos avaliar a eficácia dos herbicidas 2,4-D + picloram (Tordon), fluroxypyr + picloram (Plenum) e triclopyr (Garlon), aplicados em pulverização foliar em área total, no controle de aroeirinha e de mata-pasto em pastagem, bem como a persistência desses herbicidas no solo.

\section{MATERIAL E MÉTODOS}

O experimento foi conduzido em Viçosa, entre os meses de maio de 2003 a julho de 2004 , em pastagem estabelecida de capimgordura (Melinis minutiflora) infestada com aroeirinha (Schinus terebintifolius) e matapasto (Eupatorium maximilianii), em solo de textura argilo-arenosa, com pH 5,5 e 3\% de matéria orgânica. O delineamento experimental foi em blocos casualizados, com três repetições e 13 tratamentos, distribuídos em esquema fatorial $(3 \times 4+1)$, sendo duas misturas comerciais e um herbicida, aplicados em quatro doses mais uma testemunha. A parcela experimental possuía área de $24 \mathrm{~m}^{2}$ (4x6 m), com bordadura de $1,0 \mathrm{~m}$. As misturas comerciais utilizadas foram 2,4-D + picloram (Tordon) e fluroxypyr + picloram (Plenum) e o herbicida triclopyr (Garlon), aplicados em quatro doses (Tabela 1).

As espécies daninhas da área experimental, aroeirinha (Schinus terebintifolius) e mata-pasto (Eupatorium maximilianii), são plantas perenes e, no momento da aplicação, apresentavam-se em bom estado vegetativo; a espécie forrageira (Melinis minutiflora) encontrava-se bem estabelecida, proporcionando boa cobertura do solo.

As aplicações dos herbicidas foram feitas em ótimas condições ambientais $180 \%$ de umidade relativa do ar, temperatura de $23^{\circ} \mathrm{C}$, velocidade do vento de $4,0 \mathrm{~km} \mathrm{~h}^{-1}$ e solo úmido), utilizando um pulverizador costal, munido com barra de dois bicos de jato plano ("leque") TT 110 02, espaçados entre si de 1,0 m. Durante a aplicação manteve-se a altura da barra de $50 \mathrm{~cm}$ do alvo e a pressão constante de $210 \mathrm{kPa}$. O volume de calda aplicado foi equivalente a $100 \mathrm{~L} \mathrm{ha}^{-1}$. Foi adicionado 0,3\% de óleo Atherbane à calda aplicada. Os produtos comerciais utilizados estão especificados na Tabela 1.

Tabela 1 - Tratamentos avaliados no experimento

\begin{tabular}{|c|c|c|}
\hline Tratamento & \multicolumn{2}{|c|}{ Dose ha ${ }^{-1}$} \\
\hline Herbicida utilizado & g i.a. & Produto comercial \\
\hline$[2,4-\mathrm{D}+\text { picloram }]^{*}$ & {$[360+96]$} & 1,5 \\
\hline [2,4-D + picloram $]$ & {$[720+192]$} & 3,0 \\
\hline [2,4-D + picloram $]$ & {$[1.080+288]$} & 4,5 \\
\hline [2,4-D + picloram $]$ & {$[1.440+384]$} & 6,0 \\
\hline [fluroxypyr + picloram]** & {$[120+120]$} & 1,5 \\
\hline [fluroxypyr + picloram] & {$[240+240]$} & 3,0 \\
\hline [fluroxypyr + picloram] & {$[380+380]$} & 4,5 \\
\hline [fluroxypyr + picloram] & {$[480+480]$} & 6,0 \\
\hline Triclopyr*** & $(240)$ & 0,5 \\
\hline Triclopyr & $(480)$ & 1,0 \\
\hline Triclopyr & $(720)$ & 1,5 \\
\hline Triclopyr & $(960)$ & 2,0 \\
\hline Testemunha & 0 & 0 \\
\hline
\end{tabular}

* Tordon. ** Plenum. *** Garlon. 
As avaliações de controle das espécies daninhas foram realizadas aos 60 e 180 DAA (Dias Após a Aplicação), por meio de observações visuais, utilizando-se a escala de 0 a 100 , sendo 0 ausência de controle e 100 controle total da espécie daninha. O nível de intoxicação da pastagem foi avaliado visualmente, aos 7 e 15 DAA, utilizando-se a mesma escala para avaliação da eficiência de controle.

Aos 10, 40, 70, 120, 180 e 360 dias após aplicação (DAA) foram coletadas, aleatoriamente, em cada parcela, amostras de solo nas profundidades de 0 a 10 e 10 a $20 \mathrm{~cm}$, para condução de ensaio biológico, em condições controladas, a fim de avaliar o efeito residual dos herbicidas no solo.

Para realização do ensaio biológico em casa de vegetação utilizou-se o pepino (Cucumis sativus) como planta indicadora. Para cada amostra foram cultivadas plantas-teste em três vasos não perfurados, para evitar a lixiviação, contendo 300 g de solo, com duas plantas de pepino por vaso. Aos 30 dias após a semeadura da planta-teste foi realizada avaliação visual de intoxicação das plantas, utilizando-se a escala de 1 a 4, em que 1 representa ausência de intoxicação; 2 , intoxicação leve; 3 , intoxicação moderada; e 4, intoxicação severa (Figura 1). Essa escala foi determinada a partir de observações em experimentos preliminares, considerando-se como: ausência de intoxicação (1), plantas normais; intoxicação leve (2), plantas com sintomas de intoxicação evidentes apenas no limbo foliar, com dobramentos nos bordos (encrespamento); intoxicação moderada (3), plantas com dobramentos mais intensos nos bordos do limbo foliar e aumento de crescimento (altura), seguido de retorcimento caulinar (epinastia); e intoxicação severa (4), redução da área do limbo foliar com intensos dobramentos dos bordos, retorcimento caulinar (epinastia), seguido por engrossamento nas gemas terminais, resultando em plantas mais baixas, chegando à morte (Figura 1).

Os resultados relativos ao controle das plantas daninhas foram submetidos à análise de variância pelo teste $\mathrm{F}$, a $5 \%$ de probabilidade, sendo o efeito das doses verificado por meio de análise de regressão. As curvas de regressão foram feitas com base nas doses dos produtos comerciais, devido às misturas de herbicidas $(2,4-\mathrm{D}+$ picloram e fluroxypyr + picloram), que impossibilitam a obtenção dos modelos com a utilização dos ingredientes ativos. Para o triclopyr, embora não seja constituído de mistura, o modelo também foi obtido através das doses do produto comercial, para padronizar os resultados. Na escolha do modelo foi considerada a explicação biológica e significância do quadrado médio da regressão e das estimativas das características avaliadas. Já os resultados sobre período residual no solo foram discutidos apenas com base nos sintomas apresentados pela planta indicadora, sem a realização de análise estatística.

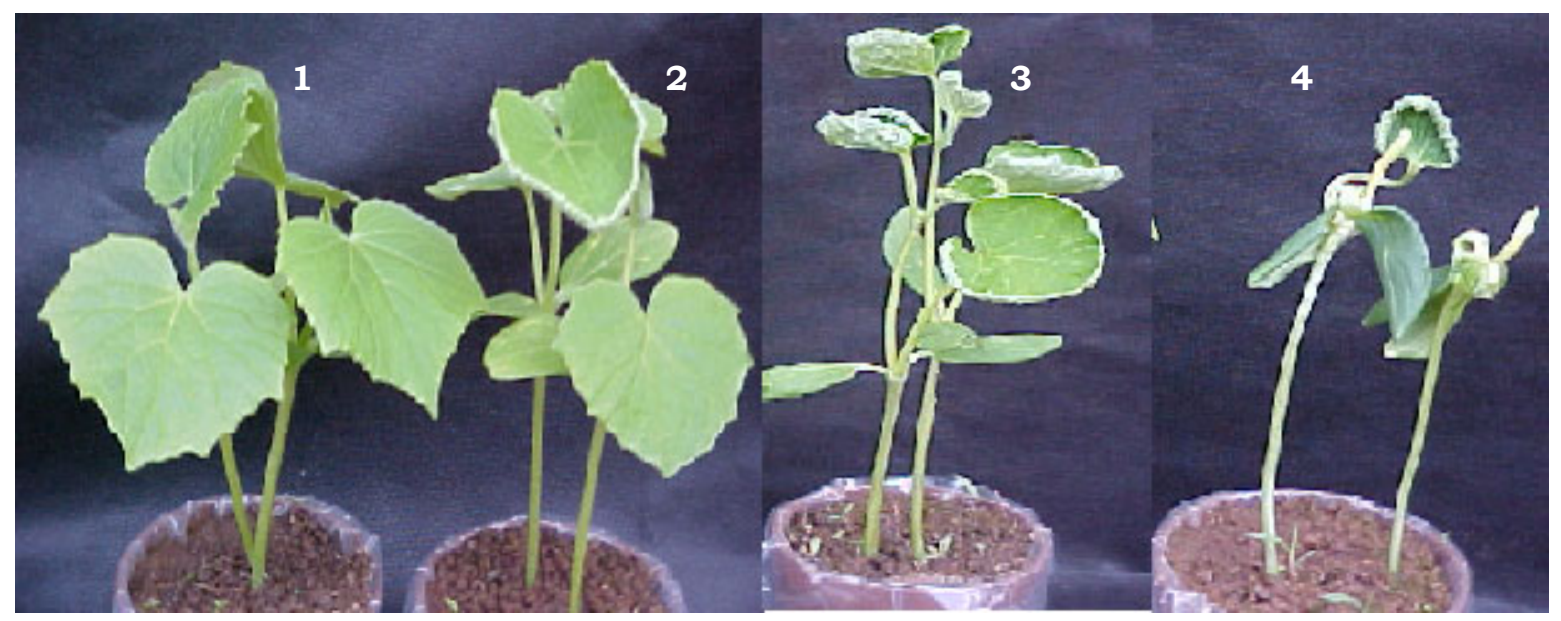

Figura 1 - Escala de intoxicação nas plantas de pepino (Cucumis sativus), em que: 1 - ausência de intoxicação; 2 - intoxicação leve; 3 - intoxicação moderada; e 4 - intoxicação severa. 


\section{RESULTADOS E DISCUSSÃO}

Nenhum dos tratamentos avaliados causou intoxicação na forrageira (Melinis minutiflora) estabelecida na pastagem.

Quanto à eficiência dos herbicidas no controle das plantas daninhas, controle superior a $90 \%$ de Schinus terebintifolius aos 60 e 180 DAA foi obtido, respectivamente, a partir de 3,72 e 2,78 $\mathrm{L} \mathrm{ha}^{-1}$ para a mistura comercial Tordon, 2,44 e 1,97 $\mathrm{L} \mathrm{ha}^{-1}$ para a mistura comercial Plenum e 0,95 e 1,23 L ha-1 para o produto comercial Garlon. Para Eupatorium maximilianii, o mesmo nível de controle, aos 60 e 180 DAA, foi obtido, respectivamente, a partir de 3,47 e 2,46 $\mathrm{L} \mathrm{ha}^{-1}$ para a mistura comercial Tordon, 2,16 e 2,02 $\mathrm{L} \mathrm{ha}^{-1}$ para a mistura comercial Plenum e 1,08 e 1,44 L ha-1 para o produto comercial Garlon (Figuras 2, 3 e 4). Freitas et al. (2003) obtiveram controle de 90\% de Desmodium incanum e Zornia latifolia em gramado infestado com Paspalum notatum, com 1,4 $\mathrm{L} \mathrm{ha}^{-1}$ de triclopyr (Garlon) e 3,0 $\mathrm{L} \mathrm{ha}^{-1}$ de 2,4-D + picloram (Tordon), sem causar nenhum dano ao gramado.

A necessidade de maior dose dos herbicidas 2,4-D + picloram (Tordon) e fluroxypyr + picloram (Plenum) aos 60 DAA, em relação aos 180 DAA, pode ser explicada segundo as características desses herbicidas. Segundo Silva et al. (2005), em geral plantas tratadas

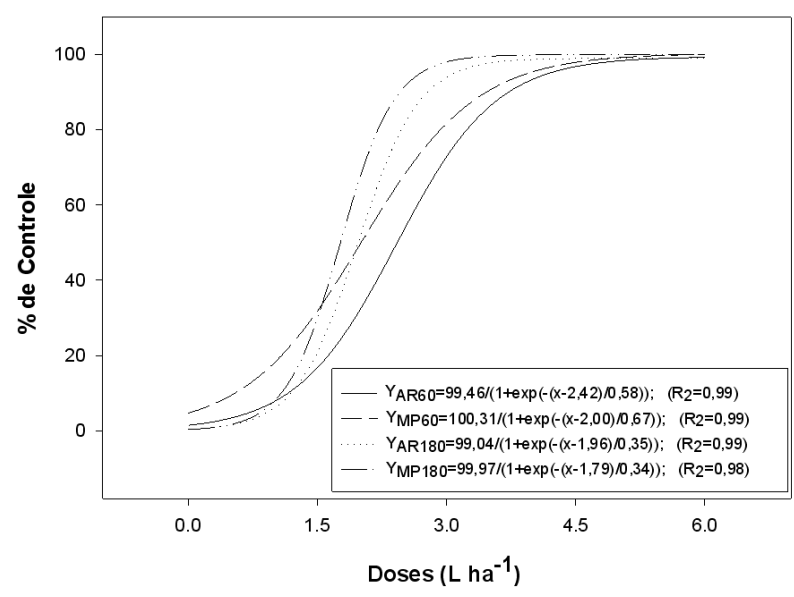

Figura 2 - Porcentagem de controle de Schinus terebintifolius (aroeirinha-AR) e Eupatorium maximilianii (mata-pastoMP) em função das doses da formulação comercial Tordon (2,4-D + picloram) aos 60 e 180 dias após a aplicação (DAA). com herbicidas hormonais ganham maior tolerância com a idade. Além disso, o picloram, na planta apresenta efeito lento, porém extremamente persistente, ou seja, a planta não consegue metabolizar rapidamente este herbicida.

Na Tabela 2, estão apresentados os resultados de intoxicação nas plantas de pepino aos $10,40,70,120,180$ e 360 DAA. Apesar de não ter ocorrido chuva no período entre a aplicação dos herbicidas e as coletas das amostras

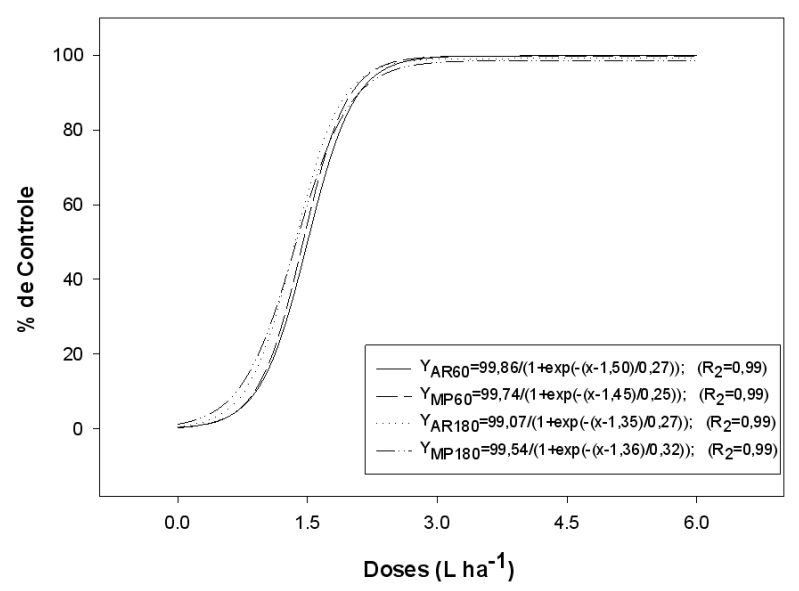

Figura 3 - Porcentagem de controle de Schinus terebintifolius (aroeirinha-AR) e Eupatorium maximilianii (mata-pastoMP) em função das doses da formulação comercial Plenum (fluroxypyr + picloram) aos 60 e 180 dias após a aplicação (DAA).

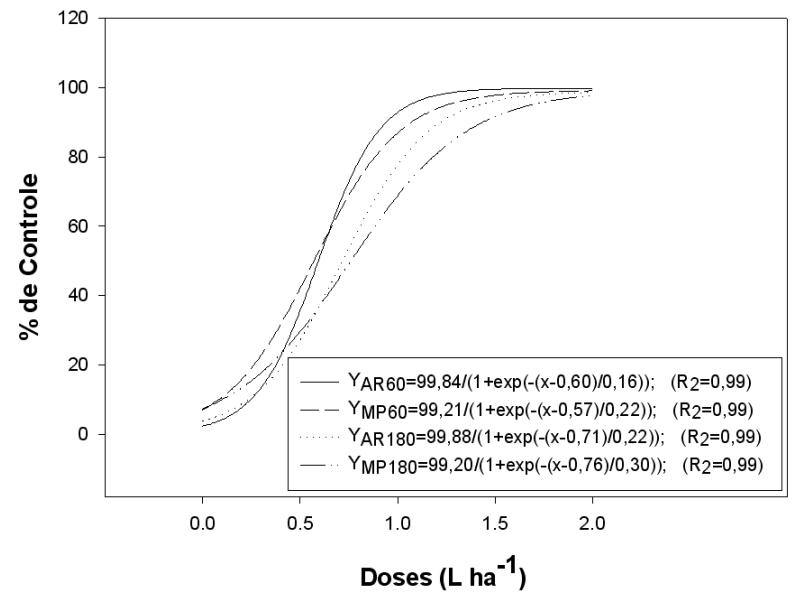

Figura 4 - Porcentagem de controle de Schinus terebintifolius (aroeirinha-AR) e Eupatorium maximilianii (mata-pastoMP) em função das doses da formulação comercial Garlon (triclopyr) aos 60 e 180 dias após a aplicação (DAA). 
aos 10 e 40 DAA, detectaram-se resíduos desses herbicidas em amostras coletadas até a profundidade de 10 a $20 \mathrm{~cm}$ (Tabela 2). Essa movimentação dos herbicidas no solo, apesar da não-ocorrência de chuva, pode ser atribuída à difusão dos herbicidas no solo e também à exsudação radicular desses produtos pelas raízes das plantas tratadas, em diferentes profundidades (Silva et al., 2005).

Resíduo de triclopyr foi observado somente nas amostras coletadas aos 10 DAA, não tendo sido observada intoxicação na planta indicadora nos tratamentos com esse produto a partir de 40 DAA (Tabela 2). Essa curta persistência de triclopyr no solo foi também relatada por Johnson et al. (1995) e Norris et al. (1987). Todavia, nas misturas comerciais utilizadas, contendo picloram, verificaram-se resíduos no solo, nas duas profundidades amostradas, até os 180 DAA.

Aos 360 DAA, o efeito residual foi verificado no solo, na profundidade de 0 a $10 \mathrm{~cm}$, para todas as doses aplicadas de fluroxypyr + picloram e a partir de $720+0,192 \mathrm{~g} \mathrm{ha}^{-1} \mathrm{de}$ 2,4-D + picloram, com leve intoxicação na planta indicadora. Nesta avaliação não foi verificado sintoma de intoxicação nas amostras coletadas na profundidade de 10 a $20 \mathrm{~cm}$ (Tabela 2). Esses resultados estão de acordo com os de MacDonald et al. (1976), os quais detectaram presença de picloram no solo por período superior a um ano após a aplicação. Estimativas da persistência de concentrações potencialmente tóxicas deste herbicida mostram variação de alguns meses até três anos, dependendo do tipo de solo e das condições ambientais (Silva, 2005; Scrifres et al., 1971; Fryer et al., 1979; Johnsen Jr., 1980; Norris et al., 1986; Neary et al., 1985; Bovey \& Richardson, 1991; Deubert \& Corte-Real, 1986; WSSA, 1994).

Apesar da alta mobilidade do picloram no solo (Inoue et al., 2003), resíduo das misturas prontas de Tordon e Plenum, ambos contendo o picloram, foi detectado aos 360 DAA apenas na camada superficial $(0$ a $10 \mathrm{~cm}$ de profundidade) (Tabela 2), possivelmente devido à retenção do herbicida na biomassa seca após a morte da planta, considerando que a aplicação foi em pós-emergência. Quando aplicada à folhagem, a maioria do picloram (70-90\%) permanece nas folhas, e apenas uma porcentagem pequena é conduzida para caules e raízes (Meikle et al., 1966; Cessna et al., 1986; Hickman et al., 1990).

Tabela 2 - Intoxicação em plantas-teste de pepino (Cucumis sativus) aos 10, 40, 70, 120, 180 e 360 DAA (dias após a aplicação), das misturas comerciais de herbicidas 2,4-D + picloram e fluroxypyr + picloram e do herbicida triclopyr, cultivados em vaso com amostras de solo retiradas nas profundidades de 0-10 e 10-20 cm no perfil do solo

\begin{tabular}{|c|c|c|c|c|c|c|c|c|c|c|c|c|}
\hline \multirow{4}{*}{ Tratamento } & \multicolumn{12}{|c|}{ ESCALA DE INTOXICAÇÃO* } \\
\hline & \multicolumn{2}{|c|}{10 DAA } & \multicolumn{2}{|c|}{40 DAA } & \multicolumn{2}{|c|}{70 DAA } & \multicolumn{2}{|c|}{120 DAA } & \multicolumn{2}{|c|}{180 DAA } & \multicolumn{2}{|c|}{360 DAA } \\
\hline & \multicolumn{12}{|c|}{ Profundidade $(\mathrm{cm})$} \\
\hline & $0-10$ & $10-20$ & $0-10$ & $10-20$ & $0-10$ & $0-10$ & $10-20$ & $10-20$ & $0-10$ & $10-20$ & $0-10$ & $10-20$ \\
\hline$[2,4-\mathrm{D}+$ picloram $]\left[360+96 \mathrm{~g} \mathrm{ha}^{-1}\right]^{* *}$ & 3 & 3 & 3 & 3 & 3 & 3 & 2 & 1 & 3 & 2 & 1 & 1 \\
\hline$[2,4-\mathrm{D}+$ picloram $]\left[720+192 \mathrm{~g} \mathrm{ha}^{-1}\right]$ & 4 & 4 & 4 & 3 & 3 & 3 & 2 & 2 & 4 & 3 & 2 & 1 \\
\hline$[2,4-\mathrm{D}+$ picloram $]\left[1080+288 \mathrm{~g} \mathrm{ha}^{-1}\right]$ & 4 & 4 & 4 & 4 & 4 & 3 & 3 & 2 & 4 & 3 & 2 & 1 \\
\hline$[2,4-\mathrm{D}+$ picloram $]\left[1440+384 \mathrm{~g} \mathrm{ha}^{-1}\right]$ & 4 & 4 & 4 & 4 & 4 & 4 & 3 & 2 & 4 & 3 & 2 & 1 \\
\hline [fluroxypyr + picloram] $\left[120+120 \mathrm{~g} \mathrm{ha}^{-1}\right]^{* * *}$ & 3 & 3 & 4 & 3 & 2 & 3 & 3 & 1 & 3 & 3 & 2 & 1 \\
\hline [fluroxypyr + picloram] $\left[240+240 \mathrm{~g} \mathrm{ha}^{-1}\right]$ & 4 & 3 & 4 & 4 & 3 & 4 & 3 & 2 & 3 & 3 & 2 & 1 \\
\hline [fluroxypyr + picloram] $\left[380+380 \mathrm{~g} \mathrm{ha}^{-1}\right]$ & 4 & 3 & 4 & 4 & 4 & 4 & 3 & 2 & 4 & 3 & 2 & 1 \\
\hline [fluroxypyr + picloram] $\left[480+480 \mathrm{~g} \mathrm{ha}^{-1}\right]$ & 4 & 4 & 4 & 4 & 3 & 4 & 3 & 2 & 4 & 3 & 2 & 1 \\
\hline triclopyr $\left(240 \mathrm{~g} \mathrm{ha}^{-1}\right)^{* * * *}$ & 2 & 2 & 1 & 1 & 1 & 1 & 1 & 1 & 1 & 1 & 1 & 1 \\
\hline triclopyr $\left(480 \mathrm{~g} \mathrm{ha}^{-1}\right)$ & 3 & 2 & 1 & 1 & 1 & 1 & 1 & 1 & 1 & 1 & 1 & 1 \\
\hline triclopyr $\left(720 \mathrm{~g} \mathrm{ha}^{-1}\right)$ & 3 & 2 & 1 & 1 & 1 & 1 & 1 & 1 & 1 & 1 & 1 & 1 \\
\hline triclopyr $\left(960 \mathrm{~g} \mathrm{ha}^{-1}\right)$ & 2 & 3 & 1 & 1 & 1 & 1 & 1 & 1 & 1 & 1 & 1 & 1 \\
\hline Testemunha & 1 & 1 & 1 & 1 & 1 & 1 & 1 & 1 & 1 & 1 & 1 & 1 \\
\hline
\end{tabular}

* Escala de intoxicação no pepino: ausência (1), leve (2), moderada (3) e severa (4). ** Tordon. *** Plenum. **** Garlon. 
A curta persistência do triclopyr no solo fica evidenciada na Figura 5, não sendo observados sintomas de intoxicação nas plantas de pepino aos 40 DAA. Contudo, para as duas misturas comerciais utilizadas confirma-se a longa persistência no solo atribuída ao picloram, já considerado em outras pesquisas (MacDonald et al., 1976; Bovey \& Richardson, 1991; Deubert \& Corte-Real, 1986; WSSA, 1994).
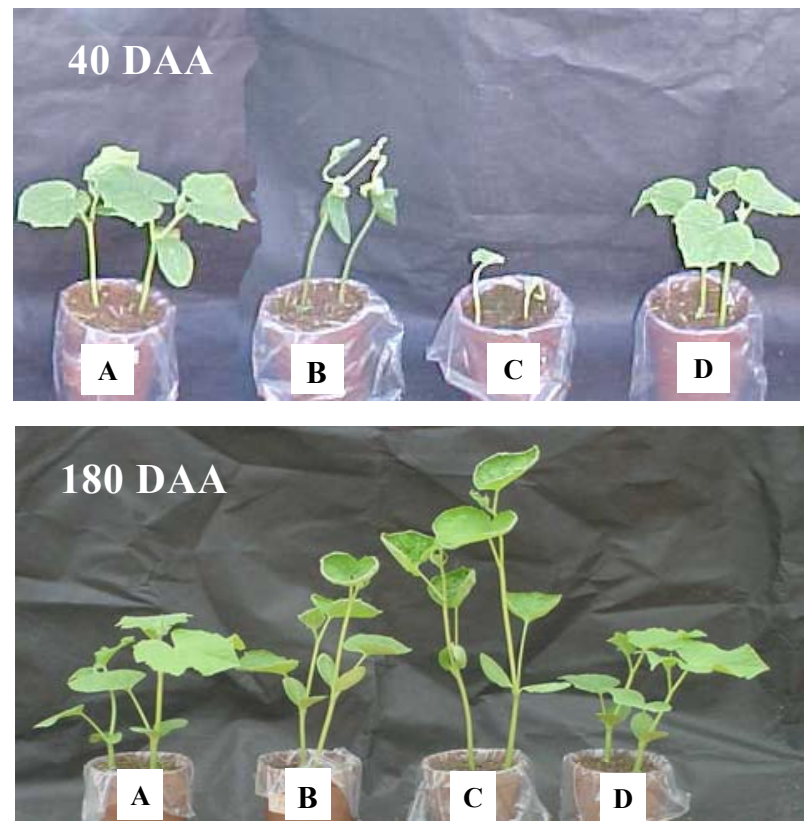

Figura 5 - Sintomas de intoxicação em plantas de pepino (Cucumis sativus) aos 40 e 180 DAA (dias após a aplicação), na profundidade de 0 a $10 \mathrm{~cm}$, para os tratamentos: (A) $480 \mathrm{~g} \mathrm{ha}^{-1}$ de triclopyr; (B) $\left[720+192 \mathrm{~g} \mathrm{ha}^{-1}\right]$ de 2,4-D + picloram; (C) $\left[240+240 \mathrm{~g} \mathrm{ha}^{-1}\right]$ de fluroxypyr + picloram; e (D) testemunha sem herbicida.

Todos os herbicidas utilizados foram eficientes no controle de Schinus terebintifolius e Eupatorium maximilianii, sem causar intoxicação na pastagem, entretanto o efeito residual no solo, nas misturas que continham picloram, foi observado até os 360 DAA. O herbicida triclopyr apresentou menor período residual no solo, sendo, portanto, para o caso, o herbicida recomendado, considerando a necessidade de se preservar o ambiente.

\section{LITERATURA CITADA}

BOVEY, R. W.; RICHARDSON, C. W. Organic chemicals in the environment. J. Environ. Qual., v. 20, p. 528-531, 1991.
CESSNA, A. J.; WADDINGTON, J.; BITTMAN, S. Residues of 2,4-D and picloram in aspen poplar and soil after application with a roller. Can. J. Plant Sci., v. 69, p. 205-212, 1986.

DEUBERT, K. H.; CORTE-REAL, I. Soil residues of picloram and triclopyr after selective foliar application on utility rights-of-way. J. Arbor., v. 12, n. 11, p. 269-272, 1986.

FREITAS, F. C. L. et al. Eficiência do triclopyr no controle de plantas daninhas em gramado (Paspalum notatum). Planta Daninha, v. 21, n. 1, p. 159-154, 2003.

FRYER, J. D.; SMITH, P. D.; LUDWIG, J. W. Long-term persistence of picloram in a sandy loam soil. J. Environ. Qual., v. 8, n. 1, p. 83-86, 1979.

HICKMAN, M. V.; MESSERSMITH, C. G.; LYM, R. G. Picloram release from leafy spurge roots. J. Range Manag., v. 43, n. 5, p. 442-445, 1990.

INOUE, M. H. et al. Critérios para avaliação do potencial de lixiviação dos herbicidas comercializados no estado do Paraná. Planta Daninha, v. 21, n. 2, p. 312-323, 2003.

JOHNSEN Jr., T. N. Picloram in water and soil from a semiarid pinyon-juniper watershed. J. Environ. Qual., v. 9, n. 4, p. 601-605, 1980.

JOHNSON, W. G.; LAVY, T. L.; GBUR, E. E. Sorption, mobility, and degradation of triclopyr and 2,4-D on four soils. Weed Sci., v. 43, p. 678-684, 1995.

MACDONALD, K.B.; MCKERCHER, R.B.; MOYER, J. R. Picloram displacement in soil. Soil Sci., v. 121, p. 94-102, 1976.

MEIKLE, R. W.; WILLIAMS, E. A.; REDEMANN, C. T. Metabolism of Tordon herbicide (4- amino-3,5,6trichloropicolinic acid) in cotton and decomposition in soil. J. Agric. Food Chem., v. 14, n. 4, p. 384-387, 1966.

NEARY, D. G. et al. Picloram movement in an Appalachian hardwood forest watershed. J. Environ. Qual., v. 14, n. 4, p. 585-592, 1985.

NORRIS, L. A. Accuracy and precision of analyses for 2,4-D and picloram in streamwater by ten contract laboratories.

Weed Sci., v. 34, p. 485-489, 1986.

NORRIS, L.; MONTGOMERY, M. L.; WARREN, L. E. Triclopyr persistence in western Oregon hill pastures. Bull. Environ. Contam. Toxic., v. 39, p. 134-141, 1987.

NYFFELER, A. et al. Collaborative studies of dose response curves obtained with diferent bioassay meted for soil-applied herbicides. Weed Res., v. 22, p. 213-222, 1982. 
OLIVEIRA JUNIOR, R. S. et al. Atividade residual de imazaquin e alchlor+atrazine para plantio seqüencial de canola. Ci. Rural, v. 31, n. 2, p. 219-224, 2001.

RODRIGUES, B. N.; ALMEIDA, F. S. Guia de herbicidas. 3.ed. Londrina: IAPAR, 2005. 591 p.

SCRIFRES, C. J. et al. Picloram persistence in semiarid rangeland soils and water. Weed Sci., v. 19, n. 4, p. 381-384, 1971.

SILVA, A. A. et al. Biologia e controle de plantas daninhas. Viçosa, MG: Universidade Federal de Viçosa, 2005. CD-Rom.

SILVA, A. A.; WERLANG, R. C.; FERREIRA, L. R. Controle de plantas daninhas em pastagens. In: OBEID J. A. et al. (Org.) SIMPÓSIO SOBRE MANEJO ESTRATÉGICO DA PASTAGEM, 2002, Viçosa-MG. Simpósio... Viçosa, MG: 2002. p. 279-310.
SOUZA, A. P. et al. Lixiviação do glyphosate e do imazapyr em solos com diferentes texturas e composição química. II Método analítico. Planta Daninha, v. 17, n. 2, p. 245-262, 1999.

THILL, D. Growth regulator herbicides. In: WELLER, S. C. et al. Herbicide action course. West Lafayette: Purdue University, 2003. p. 267-275.

WEBER, J. B.; MILLER, C. T. Organic chemical movement over and through soil. In: SAWHNEY, B. L.; BROWN, D. (Eds.). Reactions and movement of organic chemicals in soil. Madison: SSSA, 1989. p. 305-334. (SSSA Special Publication, 22).

WEED SCIENCE SOCIETY OF AMERICA - WSSA. Herbicide handbook. Champaign: 1994. 352 p. 